\title{
Network Resilience Optimisation of Complex Systems
}

\author{
Gianluca Filippi ${ }^{1[0000-0001-7393-905 X]}$ and \\ Massimiliano Vasile ${ }^{1}$ \\ James Weir Building, 75 Montrose St, Glasgow G1 1XJ \\ g.filippi@strath.ac.uk, massimiliano.vasile@strath.ac.uk
}

\begin{abstract}
This paper presents a method for the design for resilience of complex systems under uncertainty. In this approach, the complexity of the system is captured by a network formulation. Each node $i$ is completely determined by two indicators: a performance $f_{i}$ and a functionality $c_{i}$. Both these measures depend on time, decision and uncertain variables. In particular, we suggest the functional measure of resilience. The node resilience depends on the evolution over time of its state $x_{i}$ which is solution of a differential equation. Bifurcation theory is used to continuously model transitions between fully functioning and degraded states, disruptions and shocks that could affect the node.

This work shows how to exploit the network properties and to optimise the global connected resilience emerging from the coupled dynamics of the single nodes.
\end{abstract}

Keywords: Optimisation · Uncertainty · Network · Resilience.

\section{Introduction}

The design of a Complex Engineered System (CEdS) is a multi-disciplinary problem and it requires the collaboration of a high number of experts with different backgrounds. Also, particularly in the field of space engineering, the whole design process lasts for many years. These reasons suggest that lack of knowledge, conflicting opinions and subjective probability statements always heavily impact the process.

By studying accidents that happened in the past in order to develop new approaches for the risk reduction of CEdSs, it has been recognised [9] that there exists a common pattern that usually bring the CEdS to fail with possible catastrophic consequences. This pattern includes: production pressure that erodes safety margins and exposes the system to risky scenarios, the habit of taking past successes as a reason for confidence in future designs, fragmented problem solving and also problems of communication within the organisation.

The aim of this paper is to propose a new system engineering approach for the design of CEdSs that goes in the direction of solving the listed problems. 
We use a graph representation to model the CEdS and the interaction of the subsystems and components under uncertainty [3]. This approach allows to have a holistic and coherent view of the entire system and design process as well as simplifying the communications between different actors that are involved.

Traditional methods based on the estimation of margins and/or statistical moments can not be successful in modelling imprecision which is really influential particularly in the early phases of the design process.

The implementation of Imprecise Probability theories within the design methodologies represents a substantial step to solve the problem. Dempster-Shafer theory of evidence is applied in this paper.

First all the Quantities of Interest (QoIs) are identified and based on them the global indicators of performance $f$ and functionality $c$ are defined. For the latter we are interested in particular in quantifying the resilience $\rho$. The evolution of the system's state (that quantifies the resilience) is modelled with the use of Bifurcation Theory. It allows, indeed, to capture the continuous transitions between fully functioning and degraded states as well as the occurrence of disruptions and shocks that perturb the system. Such a model can also easily describe qualitative (or topological) changes in the evolution of the system state due to the uncertainty. The optimisation for resilience and the propagation of uncertainty within the optimisation process, reduce the possibility of underestimating the risk of the possible scenarios.

The proposed resilience approach combines the concepts of robustness and reliability [10]. A solution of the optimisation problem is robust if it minimise the negative effect of uncertainty on the objective function $f$. Correspondingly, from an engineering point of view, a system' design is robust if the influence of uncertainty on the performance function described by $f$ is minimised. Reliability is instead a quantity related to the constraint function $c$ and the functionality that $c$ quantify and it measures the likelihood that the item will perform its intended function for a specified time interval under stated conditions.

\section{Evidence Theory as Uncertainty Framework}

Theory of Evidence introduced by Dempster and Shafer is a generalisation of probability theory. In the latter it is required to specify the probability space $(S, \mathbb{S}, p)$. It is a triple with $S$ the set that contains everything that could occur in the particular universe under consideration, $\mathbb{S}$ a suitably restricted set of subsets of $S$ and $p$ the function that defines probability for elements of $\mathbb{S}$. Evidence Theory is instead defined by $(\Theta, \Omega, m)$. The frame of discernment $\Theta$ is the set of all the mutually exclusive and collectively exhaustive elementary events (or hypothesis) $\theta_{i}, i=1, \ldots,|\Theta|$ :

$$
\Theta=\left\{\theta_{1}, \theta_{2}, \ldots, \theta_{i}, \ldots, \theta_{|\Theta|}\right\}
$$

All the possible events (or hypotheses) could be overlapping or nested, but in the frame of discernment only the finest division of them is considered. From the 
frame of discernment one can define the power set $2^{\Theta}=(\Theta, \cup)$ by considering all possible combinations of the elements of $\Theta$ :

$$
\Omega=2^{\Theta}=\left\{\emptyset,\left\{\theta_{1}\right\}, \ldots,\left\{\theta_{|\Theta|}\right\},\left\{\theta_{1}, \theta_{2}\right\}, \ldots,\left\{\theta_{1}, \theta_{2}, \ldots, \theta_{i}\right\}, \ldots,\left\{\theta_{1}, \theta_{3}\right\}, \Theta\right\}
$$

where the generic element $\omega=\left\{\theta_{1}, \ldots, \theta_{j}\right\}$ of $\Omega=2^{\Theta}$ is a proposition that states the truth of only one of the events $\theta_{1}, \ldots, \theta_{j}$ without specifying which one.

The degree of belief, or evidence, is quantified by the bpa that assigns a value $m \in[0,1]$ to each subset of $\Omega$ :

$$
m: 2^{\Theta} \rightarrow[0,1]
$$

where the function $m$ has to satisfy the following conditions:

$$
\begin{aligned}
& m(\omega) \geq 0, \forall \omega \in \Omega \\
& m(\omega)=0, \forall \omega \notin \Omega \\
& m(\emptyset)=0 \\
& \sum_{A \in 2^{\Theta}} m(A)=1
\end{aligned}
$$

Each subset of the power set $2^{\Theta}$ with a non-zero bpa is called a Focal Element (FE) and the pair $\langle F, m\rangle$, where $F$ is the set of all $F E \mathrm{~s}$ and $m$ the corresponding bpas, is called Body of Evidence.

The Theory of Evidence requires less restrictive statements about the likelihood than the general probability theory. In particular it involves the definition of two measures: Belief and Plausibility.

For a given model of the Quantity of Interest (QoI) $f$ and the target set $\Phi$

$$
A=\{x \in \Omega \mid f(x) \in \Phi\},
$$

belief and plausibility are defined as

$$
\begin{aligned}
& \operatorname{Bel}(A)=\sum_{\omega_{i} \subseteq A} m\left(\omega_{i}\right) \\
& \operatorname{Pl}(A)=\sum_{\omega_{i} \cap A \neq \emptyset} m\left(\omega_{i}\right)
\end{aligned}
$$

\section{System Network Model}

First, the CEdS is defined as a network where each node $i \in[1, \ldots, N]$ represents a specific subsystem or discipline. 
For the generic $i$-th node, we consider then the following measures. Among all the specified requirements for the system under design, the most important one is defined as the performance indicator $f_{i}$ :

$$
f_{i}\left(\mathbf{d}_{i}^{f}, \mathbf{d}_{i, c}^{f}, \mathbf{u}_{i}^{f}, \mathbf{u}_{i, c}^{f}, \varphi_{I i}^{f}, t\right): D_{i} \times U_{i} \times T \subseteq \mathbb{R}^{m_{i}+n_{i}+1} \rightarrow \mathbb{R},
$$

while the remaining are included in the set of functionality indicators $\mathbf{c}_{i}$ :

$$
\mathbf{c}_{i}\left(\mathbf{d}_{i}^{c}, \mathbf{d}_{i, c}^{c}, \mathbf{u}_{i}^{c}, \mathbf{u}_{i, c}^{c}, \varphi_{I i}^{c}, t\right): D_{i} \times U_{i} \times T \subseteq \mathbb{R}^{m_{i}+n_{i}+1} \rightarrow \mathbb{R}^{k}
$$

In Eqs. (11) and (12) $t \in T \subset \mathbb{R}$ is the time, $\mathbf{d}_{i}$ and $\mathbf{u}_{i}$ are the set of design and uncertain variables used only within node $i$ and $\mathbf{d}_{i, c}$ and $\mathbf{u}_{i, c}$ are the set of variables shared between node $i$ and other nodes. For easiness in the notation we will omit the apex $f$ and $c$, writing $\mathbf{d}_{i}, \mathbf{d}_{i, c}, \mathbf{u}_{i}, \mathbf{u}_{i, c}, \boldsymbol{\varphi}_{I i}$ having however in mind that the couplings between nodes that define $f$ in Eq. (11) and $\mathbf{c}$ in Eq. (12) are in general different.

The global network indicators $f$ and $c$ finally arise from an emergent behaviour of the complex network. Considering a network with $N$ nodes and representing with $\mathbb{F}$ and $\mathbb{C}$ two general indicators that are problem specific, it is:

$$
\begin{aligned}
& f(\mathbf{d}, \mathbf{u})=\mathbb{F}_{i=1}^{N}\left[f_{i}\left(\mathbf{d}_{i}, \mathbf{d}_{c, i}, \mathbf{u}_{i}, \mathbf{u}_{c, i}, \boldsymbol{\varphi}_{I i}, t\right)\right] \\
& c(\mathbf{d}, \mathbf{u})=\mathbb{C}_{i=1}^{N}\left[c_{i}\left(\mathbf{d}_{i}, \mathbf{d}_{c, i}, \mathbf{u}_{i}, \mathbf{u}_{c, i}, \boldsymbol{\varphi}_{I i}, t\right)\right]
\end{aligned}
$$

Finally, based on the distinction between coupling $\left(\mathbf{u}_{c, i}\right)$ and uncoupling $\left(\mathbf{u}_{i}\right)$ variables and on the uncertainty framework given in Section 2, it is possible to distinguish the FEs that belongs to a single node (which number is $N_{F E, i}^{u}$ ) and FEs that influence more nodes (which number is $N_{F E, i}^{c}$ ).

\section{Complexity Reduction of Uncertainty Quantification}

Using the framework summarised in Section 2 it is then possible to quantify uncertainty with the measures of belief and plausibility. Dempster Shafer Theory (DST) has however a drawback due to the high computational cost for the reconstruction of the curves. Indeed an optimisation (maximisation for belief and minimisation for plausibility) is required for each FE because its worst and best case scenarios are counted in Eqs. (9) and (10).

For example, for a problem with $m$ uncertain variables, each defined over $N_{k}$ intervals, the complexity is:

$$
N_{o p t}=N_{F E}=\prod_{k=1}^{m} N_{k} .
$$

The number of FEs and consequently of optimisations is then exponential with the problem dimension. In the following, three methods are presented to reduce the cost of uncertainty quantification when DST is used. They refer to the evaluation of the Belief curve of the performance function $f$ in Eq. (11). However the generalisations to Plausibility and function $c$ in Eq. (12) are immediate. 


\subsection{Network Decomposition}

This method exploits the properties of the network representation illustrated in Section 3. We further suppose that the global indicator $f$ is a linear combination of the nodes functions $f_{i}$

$$
f(\mathbf{d}, \mathbf{u})=\sum_{i=1}^{N} f_{i}\left(\mathbf{d}_{i}, \mathbf{d}_{c, i}, \mathbf{u}_{i}, \mathbf{u}_{c, i}, \boldsymbol{\varphi}_{I i}, t\right)
$$

where this assumptions holds true in many engineering problems.

The decomposition algorithm aims at decoupling the sub-systems over the uncertain variables in order to optimise only over a small sub-set of the FEs. This procedure requires the following steps:

1. solution of the worst case scenario problem $\max _{\mathbf{u} \in U} f(\mathbf{u})$;

2. Maximisation over the coupled variables $\mathbf{u}_{c, i}$ and computation of $\operatorname{Bel}_{c, i}(A)$.

3. Maximisation over the uncoupled variables $\mathbf{u}_{i}$.

4. Reconstruction of the approximation $\widetilde{B e l}(A)$.

A detailed analysis of the approach can be found in [5]. The overall cost is

$$
N_{o p t}=N_{s} \sum_{i=1}^{m_{u}} N_{F E, i}^{u}+\sum_{i=1}^{m_{c}} N_{F E, i}^{c}
$$

where $N_{s}$ is the number of samples taken from combination of points in all the belief curves of the coupling variables $\operatorname{Bel}_{c, i}(A), N_{F E, i}^{u}$ is the number of FEs for the uncoupled variables affecting only node $i$ and $N_{F E, i}^{c}$ is the number of FEs of the coupling variables that are shared also by node $i$.

\subsection{Tree-based exploration}

In this approach the whole computation of Belief proceeds by building a tree that has at its root the whole uncertainty space with the associated global worst-case optimisation solution, and at its distal leaves the whole set of FEs, each one with an associated maximum of the quantity of interest. The heuristic that drives how the tree is built and explored is key to the rapid convergence to the correct Belief and Plausibility values. The overall procedure follows these steps:

1. solution of the worst case scenario problem $\bar{f}_{0}=\max _{\mathbf{u} \in S_{0}^{0}} f(\mathbf{u})$ where $S_{0}:=U$;

2. s-decomposition of the uncertain space in $S_{i}^{1} \cup S_{i}^{2} \cup S_{i}^{3} \ldots \cup S_{i}^{s}$ following the heuristic criterion;

3. exploration of each subdivision $S_{i}^{k}$ by the optimiser to find the worst case scenario in that macro-FE $\max _{\mathbf{u} \in S_{i}^{k}} f(\mathbf{u})$.

Points 2. and 3. are repeated recursively, where $i$ represents the iteration step, until a predefined level of accuracy or computational cost is reached. Other details of the algorithm can be found in [1]. 


\subsection{Combined Method}

A combination of the two proposed methods is finally possible in order to further reduce the overall computational cost needed to evaluate the Belief curve.

We assume here that only a particular value of the Belief is required which corresponds to a given threshold $\nu: \operatorname{Bel}(f \leq \nu)$.

The approach starts applying the tree-based algorithm. The heuristic at point 2 in Section 4.2 is based on a measure of variance of the maxima of $f$ over the FEs. In this way the uncertain space is subdivided in order to maximise the likelihood to obtain some of the maxima below $\nu$. Such subsets $S_{i}^{k}$ are then no further decomposed because their probability mass $m$ contributes entirely to $\operatorname{Bel}(f \leq \nu)$. The process is iterated until the variance drops below a specified threshold value.

At this point the set of coupling and uncoupling uncertain variables are updated removing all the FEs already evaluated and all the FEs which contribution is known to be included in the belief measure. The network decomposition of Section 4.1 is finally applied.

The use of the tree-based algorithm during the first step of the method has two major effects. First, it reduces the number of FEs that need to be explored within the following step. Furthermore, this reduction has a important impact on the network topology. The updated graph is likely to have a reduced number of links resulting in a non-linear reduction of the number of operations needed to decompose the system. In particular, if the updated network results disconnected, the decomposition approach can also be applied in parallel within each network component with a further cost reduction.

\section{Optimisation Approach}

A general formulation for the constraint optimisation under uncertainty is

$$
\begin{aligned}
& \min _{\mathbf{d} \in D} \phi(\mathbf{d}) \\
& \text { s.t. } \gamma_{j}(\mathbf{d}) \leq 0
\end{aligned}
$$

where $\phi(\mathbf{d})$ and $\gamma(\mathbf{d})$ represent the general quantification of uncertainty on functions $f$ and $\mathbf{c}$ respectively. In particular, for this quantification we choose to use DST which translates Eq. (18) to the following:

$$
\begin{aligned}
& \max _{\mathbf{d} \in D} \operatorname{Bel}\left(f(\mathbf{d}, \mathbf{u}) \leq \nu_{f}\right) \\
& \text { s.t. } \operatorname{Bel}\left(c(\mathbf{d}, \mathbf{u}) \leq \nu_{c}\right)>1-\epsilon
\end{aligned}
$$

and we want to solve Eq. (19) for fixed $\nu_{f}, \nu_{c}$ and $\epsilon$.

The method presented in Section 4.3 is used for the approximation of Belief. To further reduce the computational cost of the design process, an Efficient Global Optimisation (EGO) approach is also applied. The interested reader can find more information about EGO in [6]. 
An archive of design vectors $A_{d}$ is first generated and for each $\hat{\mathbf{d}} \in A_{d}$, $\operatorname{Bel}\left(f(\hat{\mathbf{d}}, \mathbf{u}) \leq \nu_{f}\right)$ and $\operatorname{Bel}\left(c(\hat{\mathbf{d}}, \mathbf{u}) \leq \nu_{c}\right)$ are evaluated using the complexity reduction technique presented in Section 4.3:

$$
\begin{aligned}
& \hat{\mathbf{d}} \rightarrow \operatorname{Bel}\left(f(\mathbf{d}, \mathbf{u}) \leq \nu_{f}\right) \\
& \hat{\mathbf{d}} \rightarrow \operatorname{Bel}\left(c(\mathbf{d}, \mathbf{u}) \leq \nu_{c}\right)
\end{aligned}
$$

The acquired information is used to initialise the surrogate models $\mathbb{S}_{f}$ and $\mathbb{S}_{c}$ for $f$ and $\mathbf{c}$ respectively.

The next two steps are then iterated until convergence.

A constraint maximisation over the design space D of the surrogates $\mathbb{S}_{f}$ and $\mathbb{S}_{c}$ is performed until convergence:

$$
\begin{aligned}
& \max _{\mathbf{d} \in D} \mathbb{S}_{f}(\mathbf{d}) \\
& \text { s.t. } \mathbb{S}_{c}(\mathbf{d}) \geq 1-\epsilon
\end{aligned}
$$

The design vector $\mathbf{d}^{*}$ solution of Eq. (21) is added to the archive $A_{d} . \operatorname{Bel}\left(f\left(\mathbf{d}^{*}, \mathbf{u}\right) \leq\right.$ $\left.\nu_{f}\right)$ and $\operatorname{Bel}\left(c\left(\mathbf{d}^{*}, \mathbf{u}\right) \leq \nu_{c}\right)$ are evaluated and the surrogates the surrogates $\mathbb{S}_{f}$ and $\mathbb{S}_{c}$ are finally updated.

All the design solutions in the archive $A_{d}$ are finally cross-checked with the approach in Section 4.3 and the best solution is finally selected.

\section{Resilience Framework}

In the optimisation approach described in Eq. (19), quantities $f$ and $c$ are general indicators respectively for system performance and functionality. As previously stated, we are interested in the global resilience of the CEdS. Resilience is a functional indicator of the system and then it can be incorporated within $c$ in Eq. (19). With analogy to Eqs. (11) to (14), the state indicator of each $i$-th node (sub-system) is defined by $x_{i}$ :

$$
x_{i}\left(\mathbf{d}_{i}, \mathbf{d}_{c, i}, \mathbf{u}_{i}, \mathbf{u}_{c, i}, \varphi_{I i}, t\right): D_{i} \times U_{i} \subseteq \mathbb{R}^{m+n} \rightarrow \mathbb{R},
$$

which is the solution of the dynamical model of Ordinary Differential Equation (ODE):

$$
\dot{x}_{i}=\chi_{i}\left(x_{i}, \beta_{i}\right)+\sum_{j=1}^{N} a_{x, i j} \psi_{i}\left(x_{i}, x_{j}, \beta_{i j}\right)
$$

where $a_{x, i j} \in A_{x}$ with $A_{x}$ the adjacency matrix, $\chi$ describes the self dynamics, $\psi$ the coupled dynamics between nodes and

$$
\begin{aligned}
& \beta_{i}\left(\mathbf{d}_{i}, \mathbf{d}_{c, i}, \mathbf{u}_{i}, \mathbf{u}_{c, i}\right): D_{i} \times U_{i} \subseteq \mathbb{R}^{m+n} \rightarrow \mathbb{R} \\
& \beta_{i j}\left(\mathbf{d}_{i}, \mathbf{d}_{c, i}, \mathbf{u}_{i}, \mathbf{u}_{c, i}\right): D_{i} \times U_{i} \subseteq \mathbb{R}^{m+n} \rightarrow \mathbb{R} \\
& x_{0}=\left.x\left(\mathbf{d}_{i}, \mathbf{d}_{c, i}, \mathbf{u}_{i}, \mathbf{u}_{c, i}\right)\right|_{t=t_{0}}: D_{i} \times U_{i} \subseteq \mathbb{R}^{m+n} \rightarrow \mathbb{R} .
\end{aligned}
$$

In Eq. (23) Bifurcation Theory $[2,7]$ is used to proper model the state $x_{i}$ where $\beta$ is called the bifurcation parameter and is the responsible to the possible switch 
of the node's state to different dynamical regimes. Smooth transitions involve a continuous change in the steady state of the system until the bifurcation value is crossed, giving place to a second-order phase transition. Catastrophic transitions involve a discontinuity of the steady state at the bifurcation value, giving place to first-order phase transitions.

The resilience of node $i$ is defined as the cumulative quantity:

$$
\begin{gathered}
\rho_{i}=\int_{t_{0}}^{t_{e}} x_{i} d t . \\
\rho=\mathbb{R}_{i=1}^{N}\left[\rho_{i}\right]
\end{gathered}
$$

where $\rho=1$ indicates the system fully functioning and $\rho=0$ the system with a non recoverable failure.

This description of resilience based on the dynamics of the states $x_{i}$ incorporates the concept of reliability but it is also something more. Reliability is included in the model because the global functionality given by the interactions between $x_{i}$ is considered. However the quantity $\rho$ takes into consideration both the risk of undesired scenarios due to uncertainty and also the recovery process after the scenario happened. We consider the risk to be a combination of the system's fragility and vulnerability, the former representing the ability of the system to avoid different uncertain scenario and the latter the loss quantification on the system state. Finally, the use of bifurcation theory allow to model also a continuous recovery after the loss that can bring to the same (lower or higher) state the system had before the shock. A further novelty introduced is due to the use of imprecision related to the resilience quantification. This translates to the use of the lower likelihood measure due to the ignorance affecting the problem.

\section{Application}

The proposed method is applied to the design for resilience of an observational cube-sat in Low Earth Orbit (LEO). The goal of the mission is the detection of possible fires on the Earth within a belt centred at the latitude of $50 \mathrm{deg}$. The network representation is in Fig. 2(a). Each node has been populated with analytical models that the reader can find in $[8,4]$.

The optimisation problem has been formulated as:

$$
\begin{aligned}
& \max _{\mathbf{d} \in D} \operatorname{Bel}\left(\operatorname{Mass}(\mathbf{d}, \mathbf{u}) \leq \nu_{f}\right) \\
& \text { s.t. } \operatorname{Bel}\left(\rho_{o b d h}(\mathbf{d}, \mathbf{u}, t) \leq \nu_{c}\right)>0.8
\end{aligned}
$$

The system's performance is the overall mass $f=$ Mass $=\sum M_{i}$ that is treated as objective function in the optimisation problem. The system functionality is the global network resilience $\rho$ which model the state of the On Board Data Handling $(\mathrm{OBDH})$ node during the mission time. 


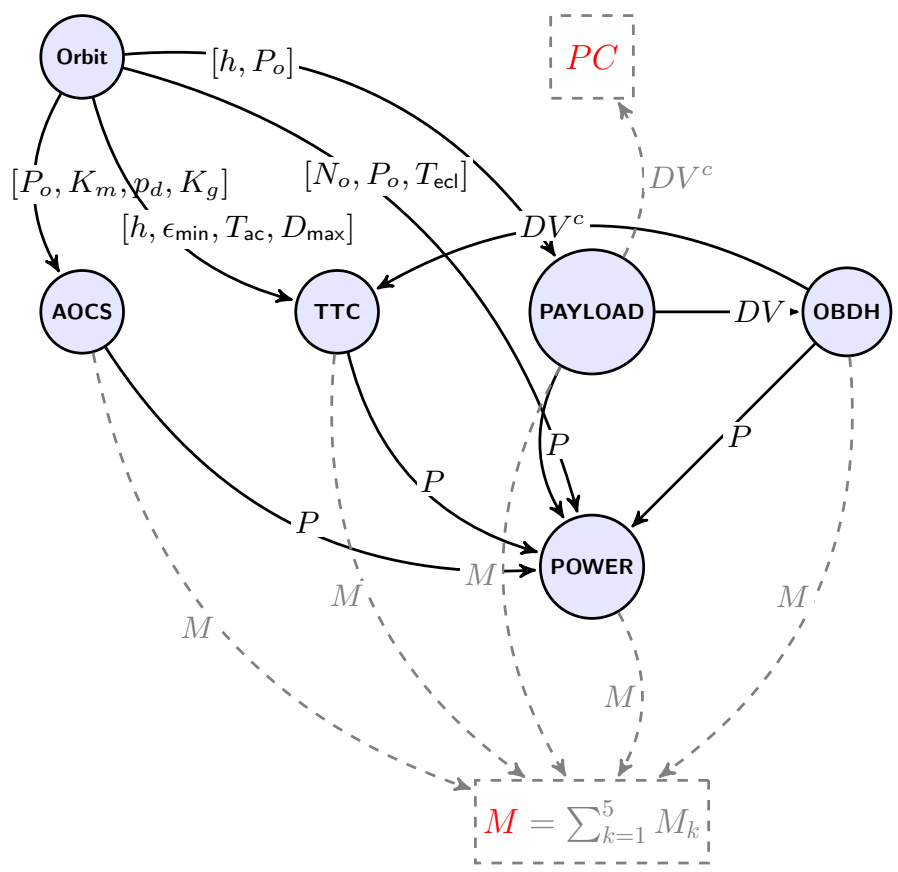

Fig. 1: Representation of the spacecraft as a complex system. The two quantities of interest are the overall mass $M$ and the percent of coverage are $P C$ for the payload.

\section{Results}

The optimisation approach in Section 5 has been applied to the test case and results are in Fig. 2. The memetic optimisation solver Multi-Population Adaptive Inflationary Differential Evolution Algorithm (MP-AIDEA) has been used that shows to be efficient and effective, on average, on a wide range of problems mixing different characteristics. Its parameters have been fixed as it follows. The number of agents for each population $N_{\text {pop }}$ and the maximum number of function evaluations for the each optimisation in the belief evaluation were set to be respectively $N_{\text {pop }}=\max \left[5, n_{D}\right]$ and $n_{\text {feval,max }}^{\text {belief }}=500 n_{U}$ with $n_{D}$ and $n_{U}$ respectively the number of design and uncertain variables while the whole process runs until convergence. The dimension of the bubble for the global restart is $\delta_{\text {global }}=0.1$, the number of populations is $n_{\text {pop }}=2$ and the convergence threshold of Differential Evolution (DE) is $\rho=0.25$.

Fig. 2(a) compares the effect of uncertainty on the system for two different design vector solution. The blue curve corresponds to the worst case optimum while the red one to the evidence-based optimisation of Eq. (27). The former shows a better worst case scenario, however, for the chosen threshold $\nu_{f}=27.5$ the latter has an higher belief to satisfy the statement. Figure Fig. 2(b) refers instead to the resilience of the solution. In Eq. (27) a threshold of 0.8 has been applied on the Belief on the resilience. For such value of belief the system is 
indeed able to recover after possible shocks due to uncertainty. However the worst case effect of the uncertainty is not recoverable as shown by the red curve.

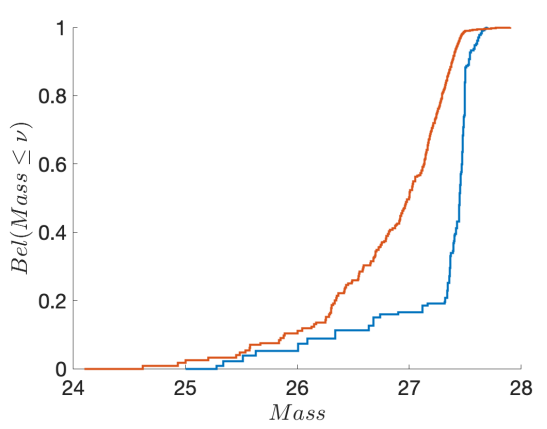

(a)

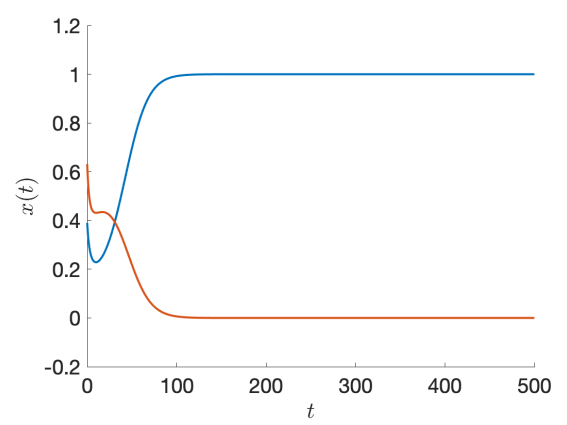

(b)

Fig. 2: (a): belief curves. The red one corresponds to the solution of min-max. The blue one to the design solution of the evidence-based optimisation (b): global network resilience for the design solution of the evidence-based optimisation. The blue curve corresponds to the threshold on the belief $\nu_{c}=0.8$ while the red one corresponds to the worst case scenario on the uncertainty space.

\section{Conclusions}

This paper presented a system engineering approach for the design optimisation of complex engineered systems. Severe uncertainty, lack of knowledge and subjective probability are important aspects to be considered during the design process. To proper model this epistemic uncertainty, it is then suggested the use of Dempster-Shafer Theory of Evidence. An approach based on Efficient Global Optimisation for the evidence-based design is suggested where a surrogate model is generated and updated during the optimisation in order to find the optimal design configuration. Three methods are further described for the computational reduction of uncertainty quantification with Evidence Theory.

A framework for the quantification of global network resilience as function of the single nodes resilience is presented and finally integrated in the optimisation algorithm.

\section{References}

1. Absil, C.O., Vasile, M.: A Variance-Based Estimation of the Resilience Indices in the Preliminary Design Optimisation of Engineering Systems Under Epistemic Uncertainty (2017) 
2. Benettin, G.: Una passeggiata tra i Sistemi Dinamici (2012)

3. Filippi, G., Vasile, M.: A Memetic Approach to the Solution of Constrained MinMax Problems. In: 2019 IEEE Congress on Evolutionary Computation, CEC 2019 - Proceedings. pp. 506-513. https://doi.org/10.1109/CEC.2019.8790124

4. Filippi, G., Vasile, M.: Global Solution of Constrained Min-Max Problems with Inflationary Differential Evolution. In: Minisci, E., Riccardi, A., Vasile, M. (eds.) Optimisation in Space Engineering OSE. No. Optimization and Engineering, Springer (2020)

5. Filippi, G., Vasile, M.: Introduction to Evidence-Based Robust Optimisation. In: Vasile, M. (ed.) Optimization Under Uncertainty with Applications to Aerospace Engineering. Springer Nature (2020)

6. Jones, D.R., Schonlau, M., Welch, W.J.: Efficient Global Optimization of Expensive Black-Box Functions. Journal of Global Optimization 13, 455-492 (1998). https://doi.org/10.1023/a:1008306431147

7. Seydel, R.: Basic Bifurcation Phenomena. Computer 49(June) (1999)

8. Wertz, James R and Larson, W.J.: Space Mission Analysis and Design. Kluwer Academic Publishers, space tech edn.

9. Woods, D.: Creating foresight: How resilience engineering can transform NASA's approach to risky decision making. Work (2), 137-144

10. Yao, W., Chen, X., Luo, W., van Tooren, M., Guo, J.: Review of uncertainty-based multidisciplinary design optimization methods for aerospace vehicles. Progress in Aerospace Sciences (6), 450-479 (aug). https://doi.org/10.1016/J.PAEROSCI.2011.05.001 